Pacific Journal of Mathematic 


\title{
SOME ERGODIC THEOREMS INVOLVING TWO OPERATORS
}

\author{
PAUL Civin
}

1. Introduction. The object of the present note is to indicate how the ergodic theorem of W. Hurewicz [3] and E. Hopf [2] can be extended to theorems involving two operators. While for a finite measure space, the Hopf theorem for two operators is readily seen to be the consequence of the theorem for one operator and the Birkhoff ergodic theorem, in the general case the theorem for two operators is established via the extended form of the Hurewicz theorem. An application is made to the theory of Markov chains in $\S 4$.

Let $(S, \Omega, \mu)$ be a fixed measure space which is assumed to be $\sigma$ finite unless otherwise stated. Capital letters are reserved for elements of $\Omega$. For a measure $\xi$ and for point functions we write $f(x)=g(x)[\xi]$ for equality almost everywhere $[\xi]$.

We consider two one-to-one transformations of $S$ onto itself, $t$ and $u$, each of which is measurable in the sense that for $v=t$ and $v=u, M \in \Omega$ implies $v M \in \Omega$ and $v^{-1} M \in \Omega$, and if $\mu(M)=0$ then $\mu\left(v^{-1} M\right)=0$. We suppose throughout that neither $t$ nor $u$ has wandering sets of positive measure, that is,

(1) For $v=t$ and $v=u$, if $A \cap v^{k} A=0, k=1,2, \cdots$, then $\mu(A)=0$.

2. The Hurewicz theorem. For any finite valued countably additive set function $\varphi$ defined on $\Omega$ and absolutely continuous with respect to $\mu$, form the set functions

$$
\varphi_{n}(X)=\sum_{k=0}^{n} \varphi\left(t^{k} X\right), \quad n=0,1, \cdots,
$$

and

$$
\nu_{n}(X)=\sum_{k=0}^{n} \mu\left(t^{k} X\right), \quad n=0,1, \cdots .
$$

Then $\varphi_{n}$ and $\nu_{n}$ are countably additive set functions and $\varphi_{n}$ is absolutely continuous with respect to $\nu_{n}$ so admits the representation

$$
\varphi_{n}(X)=\int_{X} g_{n}(x) \mu_{n}(d x), \quad n=0,1, \cdots
$$

The Hurewicz theorem then asserts that $g_{n}(x)$ has a limit at all points except for a nullset with respect to $t$, that is for all points except a $t$-invariant set of $\mu$ measure zero.

Received June 2, 1954. 
To formulate the theorem for two operators we introduce

$$
\mu_{n}(X)=\sum_{k=0}^{n} \mu\left(u^{k} X\right), \quad n=0,1, \cdots .
$$

The set function $\mu_{n}$ is countably additive but $\varphi_{n}$ is no longer automatically absolutely continuous with respect to $\mu_{n}$. In order to have this absolute continuity for any countable additive set function $\varphi$ absolutely continuous with respect to $\mu$ with the consequent representation

$$
\varphi_{n}(X)=\int_{X} f_{n}(x) \mu_{n}(d x),
$$$$
n=0,1, \cdots
$$

it is necessary for $\nu_{n}$ to be absolutely continuous with respect to $\mu_{n}$. To see this simply take $\varphi=\mu$, whence $\varphi_{n}=\nu_{n}$. We therefore take as a basic hypothesis

$$
\nu_{n} \text { is absolutely continuous with respect to } \mu_{n}
$$

with the consequent representation

$$
\nu_{n}(X)=\int_{X} c_{n}(x) \mu_{n}(d x), \quad n=0,1, \cdots
$$

We also assume that the operators $t$ and $u$ satisfy the Birkhoff ergodic theorem, that is,

(9) For $v=t$ and $v=u$, if $f(x) \in L^{1}(S), \quad \lim _{n \rightarrow \infty} \sum_{k=0}^{n} f\left(v^{k} x\right) / n$ exists almost everywhere $[\mu]$.

THEOREM 1. Let $t$ and $u$ be one-to-one measurable transformations of $S$ onto itself which have no wandering sets of positive measure. Let $\varphi$ be a finite valued countably additive set function defined on $\Omega$ and absolutely continuous with respect to $\mu$. If (7) and (9) are satisfied, then the "averaging sequence" $f_{n}(x)$ of point functions defined by (2), (5) and (6) converges everywhere except for the union of a $t$ - and u-nullset as $n \rightarrow \infty$.

Proof. We suppose first that $\mu(S)<\infty$. From the representations (4) and (8) we deduce that

$$
\varphi_{n}(X)=\int_{X} g_{n}(x) c_{n}(x) \mu_{n}(d x) .
$$

The comparison with (6) yields $f_{n}(x)=g_{n}(x) c_{n}(x)\left[\mu_{n}\right]$. The Hurewicz theorem implies that $g_{n}(x)$ has a finite limit except for a $t$-nullset. A result of C. Ryll-Nardzewski [4] shows that the hypothesis (9) that $t$ satisfies the Birkhoff ergodic theorem implies the existence of a countably additive measure $\alpha$ with the additional properties: 


$$
\begin{aligned}
& 0 \leq \alpha(X) \leq k \mu(X) . \\
& \text { lf } X=t^{-1} X, \text { then } \alpha(X)=\mu(X) . \\
& \alpha\left(t^{-1} X\right)=\alpha(X) .
\end{aligned}
$$

Likewise, since $u$ satisfies (9), there is a countably additive measure with the additional properties:

$$
\begin{aligned}
& 0 \leq \beta(X) \leq k \mu(X) \\
& \text { If } X=u^{-1} X, \text { then } \beta(X)=\mu(X) \\
& \beta\left(u^{-1} X\right)=\beta(X) .
\end{aligned}
$$

From (10.1) we note that $\alpha$ is absolutely continuous with respect to $\mu$. Hence if

$$
\alpha_{n}(X)=\sum_{k=0}^{n} \alpha\left(t^{k} X\right), \quad n=0,1, \cdots
$$

then $\alpha_{n}$ is absolutely continuous with respect to $\nu_{n}$ and we may write

$$
\alpha_{n}(X)=\int_{X} a_{n}(x) \nu_{n}(d x), \quad n=0,1, \cdots
$$

Likewise if

$$
\beta_{n}(X)=\sum_{k=0}^{n} \beta\left(u^{k} X\right), \quad n=0,1, \cdots,
$$

$\beta_{n}$ is absolutely continuous with respect to $\mu_{n}$ and

$$
\beta_{n}(X)=\int_{X} \rho_{n}(x) \mu_{n}(d x) \quad n=0,1, \cdots .
$$

If $\beta(A)=0$, then (11.3) implies $\beta\left(\bigcup_{-\infty}^{\infty} u^{k} A\right)=0$, and since $\bigcup_{-\infty}^{\infty} u^{k} A$ is $u$-invariant (11.2) implies $\mu\left(\bigcup_{-\infty}^{\infty} u^{k} A\right)=0$ and thus $\mu(A)=0$. Hence we also have the representation

$$
\mu_{n}(X)=\int_{X} b_{n}(x) \beta_{n}(d x), \quad n=0,1, \cdots .
$$

If we combine (13), (8) and (16) we obtain

$$
\alpha_{n}(X)=\int_{X} a_{n}(x) c_{n}(x) b_{n}(x) \beta_{n}(d x) .
$$

By the use of (10.3) and (11.3), (17) simplifies to

$$
\alpha(X)=\int_{X} a_{n}(x) c_{n}(x) b_{n}(x) \beta(d x), \quad n=0,1, \cdots .
$$

Since $c_{0}(x)=1[\mu]$, we find that 


$$
a_{n}(x) c_{n}(x) b_{n}(x)=a_{0}(x) b_{0}(x)[\mu], \quad n=0,1, \cdots
$$

Since we are supposing at present that $\mu(S)<\infty$, the Hurewicz theorem can be applied to (13) and (16), and thus $a_{n}(x)$ has a limit $a(x)$ as $n \rightarrow \infty$, except for a $t$-nullset and $b_{n}(x)$ has a limit $b(x)$ as $n \rightarrow \infty$, except for a $u$-nullset. By a further conclusion of the Hurewicz theorem, not already stated, we know that $a(x)$ is $t$-invariant and that

$$
\int_{X} a(x) \mu(d x)=\int_{X} a_{\vartheta}(x) \mu(d x)
$$

for every invariant set $X$. Hence for $Z=\{x \mid a(x)=0\}, \alpha(Z)=0$ and since $Z$ is $t$-invariant, $\mu(Z)=0$ by (10.2). The identical argument shows that $b(x)$ is not zero except for a $u$-nullset. If we also observe that the sets where $a_{0}(x)=\infty$ and $b_{0}(x)=\infty$ are $t$ - and $u$-nullsets respectively, as are the sets where $a_{0}(x)=0$ and $b_{0}(x)=0$, we conclude that for all $x$ except the union of a $t$ - and $u$-nullset $c_{n}(x)$ has a finite limit as $n \rightarrow \infty$. Thus $f_{n}(x)$ has a finite limit excepting the union of a $t$ - and $u$-nullset.

If the measure space $(S, \Omega, \mu)$ is $\sigma$-finite, let $k(x)$ be a bounded positive function integrable over $S$. Let

$$
\lambda(X)=\int_{X} k(x) \mu(d x)
$$

and form

$$
\lambda_{n}(X)=\sum_{j=0}^{n} \lambda\left(u^{j} X\right)
$$

The measure space $(S, \Omega, \lambda)$ is a finite measure space, and $\varphi$ is absolutely continuous with respect to $\lambda$. Hence by the first part of the proof, if

$$
\varphi_{n}(X)=\int_{X} h_{n}(x) \lambda_{n}(d x),
$$

then $h_{n}(x)$ has a finite limit at all points other then the union of a $t$ and $u$-nullset in the $\lambda$ measure and hence also in the $\mu$ measure. Thus if we let

$$
\lambda_{n}(X)=\int_{X} k_{n}(x) \mu_{n}(d x), \quad n=0,1, \cdots
$$

we have

$$
\varphi_{n}(X)=\int_{X} h_{n}(x) k_{n}(x) \mu_{n}(d x),
$$

and consequently $f_{n}(x)=h_{n}(x) k_{n}(x)\left[\mu_{n}\right]$. The Hurewicz theorem applied to (20) asserts that $k_{n}(x)$ has a finite limit except for a $u$-nullset, which implies the conclusion of the theorem. 
THEOREM 2. If in addition to the hypotheses of Theorem $1 \mu(S)<\infty$ and $t$ and $u$ commute, then $f(x)=\lim _{n \rightarrow \infty} f_{n}(x)$ has the properties

(i) $f(t x)=f(x)$

(ii) $\int_{X} f(x) \mu(d x)=\int_{X} f_{0}(x) \mu(d x)$ for any t-invariant set $X$.

Proof. We use the same notation as in the proof of Theorem 1. From (10.1) we see that any function integrable with respect to $\alpha$ is also integrable with respect to $\mu$. Hence the counterpart of (9) is satisfied with $v=u$ and $\mu$ replaced by $\alpha$. By a further use of the results of C. Ryll-Nardzewski we find the existence of a countably additive measure $\gamma$, defined as a Banach-Mazur limit

$$
\gamma(X)=\lim _{n \rightarrow \infty} \frac{1}{n+1} \sum_{j=0}^{n}\left(u^{-j} X\right)
$$

and having the additional properties:

$$
\begin{aligned}
& 0 \leq \gamma(X) \leq k_{1} \alpha(X) \\
& \text { If } X=u^{-1} X, \gamma(X)=\alpha(X) \\
& \gamma\left(u^{-1} X\right)=\gamma(X) .
\end{aligned}
$$

Since $\alpha$ is $t$-invariant and $t$ and $u$ commute we have $\alpha\left(u^{-j} t X\right)=$ $\alpha\left(u^{-j} X\right)$, and thus the definition of $\gamma(X)$ shows

$$
\gamma(X)=r\left(t^{-1} X\right)
$$

We similarly obtain a countably additive measure with the properties:

$$
\begin{aligned}
& 0 \leq \delta(X) \leq k_{1} \beta(X) \\
& \text { If } X=t^{-1} X, \text { then } \delta(X)=\beta(X) \\
& \delta\left(t^{-1} X\right)=\delta(X) \\
& \delta\left(u^{-1} X\right)=\delta(X) .
\end{aligned}
$$

From (21.1) we obtain

$$
\gamma(X)=\int_{X} m(x) \alpha(d x) .
$$

An earlier argument showed that $\delta(X)=0$ implies $\beta(X)=0$, hence

$$
\beta(X)=\int_{X} n(x) \delta(d x) .
$$

The combination of (23), (18), (19) and (24) then yields

$$
\gamma(X)=\int_{X} m(x) a_{0}(x) b_{0}(x) n(x) \delta(d x) .
$$


Since $\gamma$ and $\delta$ are both $t$ - and $u$-invariant, the integrand must be both $t$ - and $u$-invariant. With the aid of (10.2), (11.2), (21.2) and (22.2) it is then seen that $m(x) a_{0}(x) b_{0}(x) n(x)=1[\delta]$.

Likewise the $t$-invariance of $\gamma$ and $\alpha$ shows that $m(x)=1[\alpha]$, and the $u$-invariance of $\beta$ and $\delta$ shows that $n(x)=1[\beta]$. Since a set of measure zero in any of the measures $\alpha, \beta, \delta$, and $\mu$ is also of measure zero in any of the other measure, we conclude that $a_{0}(x) b_{0}(x)=1[\mu]$.

The Hurewicz theorem, applied to (13), implies that for any $t$ invariant set $X$, if we let $a(x)=\lim _{n \rightarrow \infty} a_{n}(x)$

$$
\int_{X} a(x) \mu(d x)=\int_{X} a_{0}(x) \mu(d x)=\alpha(X) .
$$

If we combine (10.2) with (25) we find

$$
\mu(X)=\int_{X} a(x) \mu(d x) .
$$

The $t$-invariance of $a(x)$ then yields $a(x)=1[\mu]$. A repetition of the argument shows that $\lim _{n \rightarrow \infty} b_{n}(x)=1[\mu]$, consequently $\lim _{n \rightarrow \infty} c_{n}(x)=1[\mu]$. The conclusions of the theorem now follow from the corresponding conclusions of the Hurewicz theorem applied to (4).

\section{The Hopf theorem.}

THEOREM 3. Let $t$ and $u$ be one-to-one measure preserving transformations of $S$ onto itself. Let $f(x) \in L^{1}(S)$ and $g(x)>0$, then for almost all $x$ the quotient

$$
\sum_{j=0}^{n} f\left(t^{j} x\right) / \sum_{j=0}^{n} g\left(u^{j} x\right)
$$

has a limit as $n \rightarrow \infty$.

Proof. Let

$$
\lambda(X)=\int_{X} g(x) \mu(d x), \quad \lambda_{n}(X)=\sum_{j=0}^{n} \lambda\left(u^{j} X\right), \quad \rho_{n}(X)=\sum_{j=0}^{n} \lambda\left(t^{j} X\right) .
$$

Then $\rho_{n}$ is absolutely continuous with respect to $\lambda_{n}$ and

$$
\varphi(X)=\int_{X} f(x) \lambda(d x)
$$

is a finite valued countably additive set function absolutely continuous with respect to $\lambda$. We form $f_{n}(x)$ according to (4) and (5) with $\mu$ replaced by $\lambda$. Now

$$
\lambda_{n}(X)=\int_{X} \sum_{j=0}^{n} g\left(u^{j} x\right) \mu(d x),
$$


so

$$
\varphi_{n}(X)=\int_{X} f_{n}(x) \lambda_{n}(d x)=\int_{X} f_{n}(x) \sum_{j=0}^{n} g\left(u^{j} x\right) \mu(d x) .
$$

But by definition

$$
\varphi_{n}(x)=\int_{X} \sum_{j=0}^{n} f\left(t^{j} x\right) \mu(d x)
$$

Thus

$$
f_{n}(x)=\sum_{j=0}^{n} f\left(t^{j} x\right) / \sum_{j=0}^{n} g\left(u^{j} x\right)
$$

and the conclusion follows from Theorem 1.

4. An application. In a recent note [1] T. E. Harris and Herbert Robbins used the Hopf ergodic theorem to obtain results concerning Markov chains admitting an infinite invariant measure. We indicate below the corresponding results that are obtainable by the use of Theorem 3.

Consider the real valued Markov chain $\cdots, x_{-1}, x_{0}, x_{1}, \cdots$ with a stationary transition probability function

$$
h(u, B)=\operatorname{prob}\left(x_{n+1} \in B \mid x_{n}=u\right) .
$$

It is assumed that there is a measure II on the real Borel sets, which does not vanish identically, is finite for bounded Borel sets and satisfies

$$
\Pi(B)=\int_{-\infty}^{\infty} h(u, B) \Pi(d u) \text {. }
$$

Let $\Phi$ be the class of real Borel sets, $S$ the space of sequences of real numbers $x=\left(\cdots, x_{-1}, x_{0}, x_{1}, \cdots\right)$ and $\Omega$ the Borel extension of the cylinder sets, in $S$. If $A \subseteq \Omega$ is determined by the coordinates $x_{k}, x_{k+1}$, $\cdots, x_{r}$ then $q\left(A \mid x_{k}=u\right)$ will denote the probability of $A$ relative to the Markov chain starting with $x_{k}=u$, as specified by $h$.

A measure is established [1] in $\Omega$ by the relation

$$
m(A)=\int q\left(A \mid x_{j}=u\right) \amalg(d u)
$$

for cylinder sets determined by $x_{k}, \cdots, x_{r}$.

We shall apply Theorem 3 , with $t$ the ath shift transformation, $(t x)_{i}=x_{i+a}$, and $u$ the $b$ th shift transformation. If $\Gamma \in \Phi$, let $R_{\Gamma}$ be the event that $x_{n} \in \Gamma$ infinitely often. The assumption

$$
\text { If } \Gamma \in \Phi \text {, then } q\left(R_{\Gamma} \mid x_{0}=u\right)=1[11],
$$

then yields [1] that $t$ and $u$ are $m$ measure preserving and that neither $t$ nor $u$ has wandering sets of positive measure. 
THEOREM 4. If (26) is satisfied, $h(u)$ is II summable and $k(u)>0$, then for almost all $x_{0}[\mathrm{II}]$

$$
\lim _{n \rightarrow \infty} \frac{h\left(x_{c}\right)+h\left(x_{a+c}\right)+\cdots+h\left(x_{n c+c}\right)}{k\left(x_{d}\right)+h\left(x_{b+d}\right)+\cdots+k\left(x_{n b+d}\right)}
$$

exists with probability one.

THEOREM 5. Let $y_{1}, y_{2}, \cdots$ be independent random variables with a common distribution function. Suppose that for any interval I

$$
\operatorname{prob}\left(y_{c}+y_{a+c}+\cdots+y_{n a+c} \in I \text { infinitely often }\right)=1
$$

and

$$
\operatorname{prob}\left(y_{a}+y_{b+a}+\cdots+y_{n b+a} \in I \text { infinitely often }\right)=1 \text {. }
$$

Then for $h(u)$ Lebesgue integrable $k(u)>0$ and almost all $m$

$$
\lim _{n \rightarrow \infty} \frac{\sum_{p=0}^{n} h\left(m+\sum_{j=0}^{n} y_{j a+c}\right)}{\sum_{p=0}^{n} k\left(m+\sum_{j=0}^{n} y_{j b+a}\right)}
$$

exists with probability one.

\section{REFERENCES}

1. T. E. Harris and H. Robbins, Ergodic theory of Markov chains admitting an infinite invariant measure, Proc. Nat. Acad. Sci., 39 (1953), 860-864.

2. E. Hopf, Ergodentheorie, Berlin 1937.

3. W. Hurewicz, Ergodic theorem without invariant measure, Ann. Math., 45 (1944), 192-206.

4. C. Ryll-Nardzewski, On the ergodic theorem I, Studia Math., 12 (1951), 65-73.

UNIVERSITY OF OREGON AND

The Institute For AdVAnCEd Study 
.././. ./FrontMatter/paper .pdf 


\section{Pacific Journal of Mathematics}

Nesmith Cornett Ankeny and Theodore Joseph Rivlin, On a theorem of S.

Bernstei........................................ 849

Louis Auslander, The use of forms in variational calculation .......... 853

Paul Civin, Abstract Riemann sum . .......................... 861

Paul Civin, Some ergodic theorems involving two operator ............ 869

Eckford Cohen, The number of solutions of certain cubic congruence .... . 877

Richard M. Cohn, Specializations over difference field .............. 887

Jean Dieudonné, Pseudo-discriminant and Dickson invarian . . ......... 907

Ky Fan, A comparison theorem for eigenvalues of normal matrice ........ 911

Richard P. Gosselin, On the convergence behaviour of trigonometric interpolating polynomial ........................... 915

Peter K. Henrici, On generating functions of the Jacobi polynomial . . . . . . . 923

Meyer Jerison, An algebra associated with a compact grou ............ 933

Wilhelm Magnus, Infinite determinants associated with Hill's equatio . . . . . 941

G. Power and D. L. Scott-Hutton, The slow steady motion of liquid past a semi-elliptical bos................................. 953

Lyle E. Pursell, An algebraic characterization of fixed ideals in certain function ring .................................... 963

C. T. Rajagopal, Additional note on some Tauberian theorems of O. Szás . . 971 Louis Baker Rall, Error bounds for iterative solutions of Fredholm integral

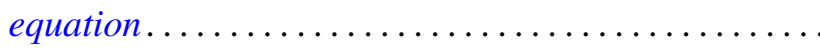

Shigeo Sasaki and Kentaro Yano, Pseudo-analytic vectors on

pseudo-Kählerian manifold ......................

Eugene Schenkman, On the tower theorem for finite group

P. Stein and John E. L. Peck, On the numerical solution of Poisson's equation over a rectangl ........................

Morgan Ward, The mappings of the positive integers into themselves which preserve divisio .

Seth Warner, Weak locally multiplicatively-convex algebra 1025

Louis Weisner, Group-theoretic origin of certain generating function .... 